\title{
Angioimmunoblastic T-Cell Lymphoma Mimicking Drug Reaction with Eosinophilia and Systemic Symptoms (DRESS Syndrome)
}

\author{
Joanna Mangana ${ }^{a} \quad$ Emmanuella Guenova $^{a, b} \quad K^{a t r i n ~ K e r l ~}{ }^{a}$ \\ Mirjana Urosevic-Maiwald ${ }^{a}$ Valerie C. Amann ${ }^{a, c}$ Cornelia Bayard $^{d}$ \\ Reinhard Dummer ${ }^{a}$ Lars E. French ${ }^{a}$ \\ ${ }^{a}$ Department of Dermatology, University Hospital Zurich, Zurich, Switzerland \\ ${ }^{b}$ Department of Dermatology, Cantonal Hospital St. Gallen, St. Gallen, Switzerland; \\ 'Department of Internal Medicine, Cantonal Hospital Aarau, Aarau, Switzerland; \\ ${ }^{\mathrm{d}}$ Department of Internal Medicine, University Hospital Zurich, Zurich, Switzerland
}

\section{Keywords}

Angioimmunoblastic T-cell lymphoma - DRESS syndrome · Drug reaction with eosinophilia and systemic symptoms

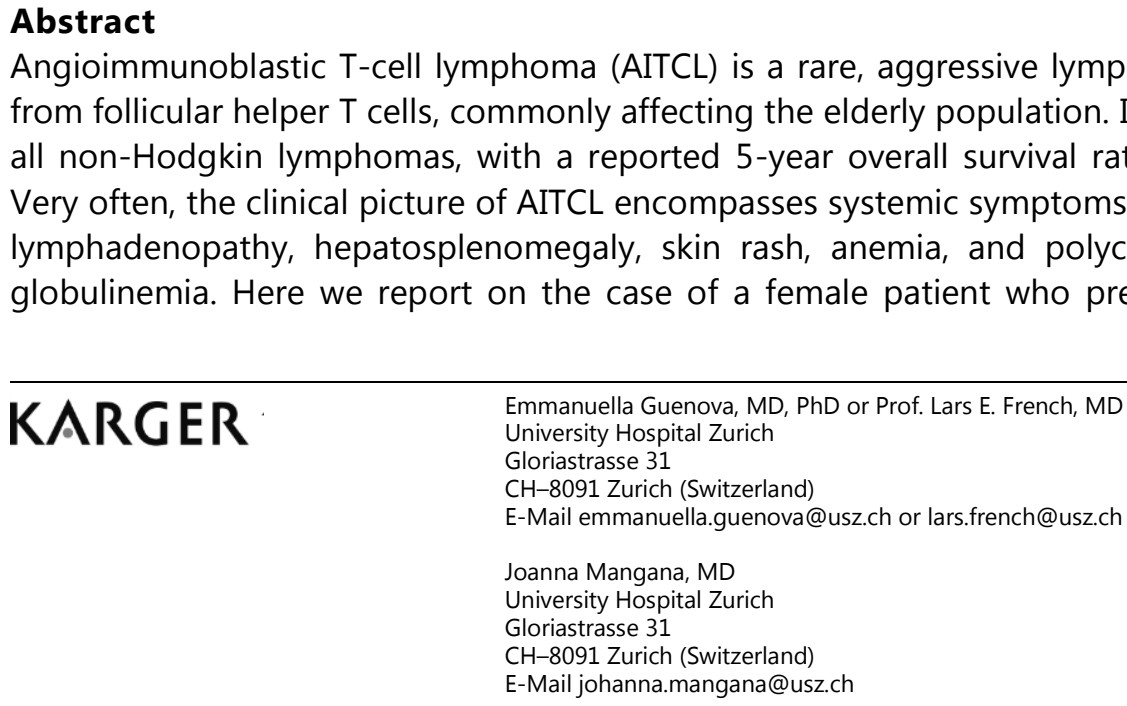

Angioimmunoblastic T-cell lymphoma (AITCL) is a rare, aggressive lymphoma which derives from follicular helper T cells, commonly affecting the elderly population. It accounts for $2 \%$ of all non-Hodgkin lymphomas, with a reported 5-year overall survival rate of less than $30 \%$. Very often, the clinical picture of AITCL encompasses systemic symptoms such as generalized lymphadenopathy, hepatosplenomegaly, skin rash, anemia, and polyclonal hypergammaglobulinemia. Here we report on the case of a female patient who presented with clinical 
features resembling drug reaction with eosinophilia and systemic symptoms (DRESS syndrome) prior to the definitive diagnosis of AITCL. The index of suspicion for cutaneous manifestations of lymphoma, and especially AITCL, must be high, particularly in atypical clinical courses of drug eruptions or if skin lesions relapse and are refractory to standard high-dose systemic corticosteroids.

(C) 2017 The Author(s)

Published by S. Karger AG, Basel

\section{Case Report}

A 65-year-old, otherwise healthy female patient presented to our emergency department with an erythematous, confluent, itchy, maculopapular exanthema on her trunk and extremities which had started 7 days before admission. In addition, she complained of a gradual onset of malaise, weight loss, arthralgia, and night sweats, having lasted about 3 weeks. The initial diagnosis was parainfectious exanthema. Topical and systemic steroids (prednisone $50 \mathrm{mg}$ ) were prescribed and the patient was discharged. Four days later, she revisited our department with facial edema and with the exanthema now having spread all over her body. On admission, she was afebrile with normal vital signs. A detailed medication history was obtained. The patient confirmed having taken diclofenac once and stimulant laxatives 5 months and 6 weeks before the onset of exanthema, respectively. Furthermore, local dental anesthesia had been performed 2 weeks before symptom onset, and she had been on St. John's wort (Hypericum perforatum) for minor depression for 1 year.

A physical examination revealed cervical lymphadenopathy, splenomegaly, and suberythroderma (Fig. 1). Laboratory tests showed mild leukocytosis (10.65 G/L) with atypical lymphocytes in the peripheral blood smear, mild anemia (Hb $116 \mathrm{~g} / \mathrm{L})$, eosinophilia (1.20 G/L), and mildly elevated AST (40 U/L) and creatinine levels $(83 \mu \mathrm{mol} / \mathrm{L})$. The patient was lymphopenic $(0.58 \mathrm{G} / \mathrm{L})$ and had normal platelet count $(392 \mathrm{G} / \mathrm{L})$. Her lactate dehydrogenase levels were normal. Epstein-Barr virus (EBV) PCR (3,316 copies $/ \mathrm{mL}$ ) and EBV serology were positive (Epstein-Barr nuclear antigen IgG and EBV IgG positive; EBV IgM negative). Human herpes virus (HHV)-6 and -8 were not reactivated, and HIV and HBV/HCV were negative. Serum protein electrophoresis, immunofixation, and serum free light chains were unremarkable. Chest radiography showed mild mediastinal widening. An abdominal ultrasound confirmed splenomegaly $(13.7 \mathrm{~cm}$ diameter) and showed retroperitoneal lymphadenopathy. A skin biopsy, performed at presentation, revealed spongiotic dermatitis with perivascular lymphocytic infiltration with eosinophils (Fig. 2a). Histologically, eczema versus eczematous drug reaction was discussed. No signs of atypical lymphocytic infiltration were noted.

In view of the exanthema, eosinophilia, facial edema, lymphadenopathy with systemic involvement, and medication history, as well as taking into account the histology report, a diagnosis of drug reaction with eosinophilia and systemic symptoms (DRESS syndrome) with an atypical reactivation of EBV was proposed. Diclofenac and articaine were considered as possible causative medications. In support of this, a lymphocyte transformation test detected T-cell sensitization against diclofenac. The patient was treated with intravenous corticosteroids followed by oral prednisone $(1 \mathrm{mg} / \mathrm{kg})$ upon improvement. Topical emollients to relieve the skin tenderness were applied.

Despite clinical improvement upon treatment, her originally reported weight loss and night sweats remained, while the skin lesions and lymphadenopathy became progressively severe. The patient was readmitted to our clinic, where another skin biopsy was performed, and the histological findings were similar to the previous ones, again in support of a drug 
reaction (Fig. 2b). However, the course of the disease, as well as thoracic and abdominal CT scans, revealed generalized lymphadenopathy and splenomegaly $(16.4 \mathrm{~cm})$, which directed our suspicion towards a hematological disease. An inguinal lymph node excision biopsy was conducted, showing lymphocyte hyperplasia with effacement of the normal architecture and high vascular proliferation with an increased number of $\mathrm{CD} 23^{+}$follicular dendritic cells. The cellular infiltrate consisted of a monomorphic, small- to medium-sized population of lymphocytes $\mathrm{CD}^{+} / \mathrm{CD}^{+}$with coexpression of programmed cell death protein 1 (PD1), and of abundant lymphoid cells partly with a blastic morphology and plasma cell differentiation. Expression of CD79a and, partially, CD20 for the B cells and of CD30 for the large lymphoid blasts was noted. The atypical cells were negative for anaplastic lymphoma kinase. EpsteinBarr encoding region (EBER) in situ hybridization revealed EBER-positive nuclei, and our patient was finally diagnosed with angioimmunoblastic T-cell lymphoma (AITCL). Her bone marrow revealed no further pathological findings.

The patient was subsequently referred to the hemato-oncology unit for further treatment. She underwent CHOP (cyclophosphamide-hydroxydaunorubicin-oncovin-prednisone) chemotherapy followed by autologous stem cell transplantation. Unfortunately, 3 months after stem cell transplantation her disease rapidly progressed and she was put on IGEV (ifosfamide, gemcitabine, and vinorelbine) salvage chemotherapy. At the last follow-up, the patient was in a poor general condition, and she was admitted to a palliative care unit, where she deceased few days afterwards.

\section{Discussion}

AITCL is a rare, aggressive lymphoma which derives from follicular T-helper cells of the germinal center of the secondary lymphoid organs, commonly affecting the elderly population, with a reported 5-year overall survival rate of less than 30\% [1-4]. Specific cutaneous infiltrates can be the first manifestation of AITCL; however, nonspecific skin manifestations such as maculopapular exanthema, purpura, and erythroderma occur in approximately half of the patients [5]. In more than one-third of these cases, a drug - mostly an antibiotic - has been chronologically associated with the onset of the disease. Furthermore, it has been associated with several viral infections such as tuberculosis, Cryptococcus neoformans infection, and lymphotropic virus infection. The best evidence currently available implicates EBV in its pathogenesis, since EBV-infected cells have been found in nearly all AITCL patients. This suggests a dynamic interplay, indicating either that the viral infection plays an active role in the pathogenesis and progression of AITCL or that the viral infection is secondary due to immune dysregulation during disease progression [6]. Also in our patient, EBER in situ hybridization was positive. The serology showed positive IgG, positive Epstein-Barr nuclear antigen IgG, negative IgM, and a positive PCR, a constellation indicative of immune suppression.

In this context, DRESS syndrome, a T-cell-mediated, delayed-type IV, potentially lifethreatening hypersensitivity reaction with maculopapular exanthema, fever, internal organ involvement, atypical lymphocytes in peripheral blood, and generalized lymphadenopathy, poses a challenging differential diagnosis to AITCL or other lymphoproliferative disorders. Even monoclonal T-cell receptor rearrangement has been reported in drug reactions, rendering the diagnosis of DRESS syndrome versus lymphoma more difficult [7]. On the other hand, benign reactive lymphoproliferative disorders such as Kikuchi disease or infectious mononucleosis are sometimes initially misdiagnosed as lymphoma. Interestingly, over the 
last years an association of DRESS syndrome with viral reactivation of herpes family members (e.g., HHV-6, HHV-7, EBV, and CMV) induced by the causative drug has been demonstrated in the majority of DRESS syndrome patients, complicating the diagnostic process even more - at least in early disease stages [8].

Our patient fulfilled the proposed diagnostic criteria for DRESS syndrome: rash, eosinophilia, and atypical lymphocytes, as well as mild nephritis and hepatitis. Although the histology was not specific, showing mainly spongiotic changes with exocytosis of lymphocytes, together with the clinical features it was compatible with a spongiotic drug reaction, one of the possible histological manifestations of DRESS syndrome. Moreover, the presence of atypical lymphocytes is a feature of DRESS syndrome, even if it is also likely to be explained in the course of EBV reactivation.

The classic drugs causative of DRESS syndrome are anticonvulsants, allopurinol, sulfonamides, antibiotics, and nonsteroidal anti-inflammatory drugs (NSAIDs). Since our patient had taken NSAIDs before the symptoms' onset, and due to a positive lymphocyte transformation test, we initially diagnosed her with DRESS syndrome caused by NSAIDs. Unique to DRESS syndrome is its late onset in relation to the period of introduction of the causative drug, which can occur around 3 weeks to 3 months previously, as in our case, and the possible persistence or worsening of the symptoms despite withdrawal of the offending drug. It has been shown that patients with severe systemic involvement have higher numbers of CD8+ lymphocytes secreting TNF- $\alpha$, IFN- $\gamma$, and IL-2 than those with mild symptoms, which can remain for more than 3 months, explaining the usually prolonged course of DRESS syndrome after drug withdrawal [8].

However, our patient's symptoms did not improve after the administration of systemic steroids, which is why she underwent a further diagnostic evaluation with an excisional lymph node biopsy, the gold standard for the diagnosis of AITCL. In hindsight, our patient's polyarthralgia was likely a paraneoplastic manifestation of AITCL, while the weight loss, fever, and night sweats were typical B-type symptoms.

Recently it has been demonstrated that patients with AITCL harbor a distinct $\mathrm{CD}^{+} / \mathrm{CD}^{+} / \mathrm{PD}^{+} / \mathrm{CXCL} 3^{+} / \mathrm{CD} 10^{+}$follicular $\mathrm{T}$-helper cell population, which, together with the detection of a network of $\mathrm{CD}_{23} 3^{+}$follicular dendritic cells and $\mathrm{EBER}^{+}$atypical $\mathrm{B}$ cells, is specific to the diagnosis [9].

There is no standard treatment for AITCL. Anthracycline-based therapeutic regimens have elicited limited responses. However, strategies targeting the NF- $\kappa B$ pathway and CD52 have held some promise. Recently, new insights were gained in the pathobiology and classification of AITCL using gene expression profiling, opening the field for possible targeted therapies [10].

In conclusion, findings of an atypical clinical course of drug eruptions, especially DRESS syndrome, or a poor response to high-dose systemic corticosteroids, as noted in our case, should raise suspicion for underlying AITCL, and further evaluation is highly recommended.

\section{Acknowledgement}

We are indebted to the patient and her family. 


\section{Statement of Ethics}

Informed consent was obtained from the patient prior to publication.

\section{Disclosure Statement}

The authors declare no conflicts of interest.

\section{References}

1 Federico M, Rudiger T, Bellei M, Nathwani BN, Luminari S, Coiffier B, Harris NL, Jaffe ES, Pileri SA, Savage KJ, Weisenburger DD, Armitage JO, Mounier N, Vose JM: Clinicopathologic characteristics of angioimmunoblastic T-cell lymphoma: analysis of the International Peripheral T-Cell Lymphoma Project. J Clin Oncol 2013;31:240-246.

2 Swerdlow SH, Campo E, Pileri SA, Harris NL, Stein H, Siebert R, Advani R, Ghielmini M, Salles GA Zelenetz AD, Jaffe ES: The 2016 revision of the World Health Organization classification of lymphoid neoplasms. Blood 2016;127:2375-2390.

3 de Leval L, Parrens M, Le Bras F, Jais JP, Fataccioli V, Martin A, et al: Angioimmunoblastic T-cell lymphoma is the most common T-cell lymphoma in two distinct French information data sets. Haematologica 2015;100:e361-e364.

4 Ganesan TS, Dhaliwal HS, Dorreen MS, Stansfeld AG, Habeshaw JA, Lister TA: Angio-immunoblastic lymphadenopathy: a clinical, immunological and molecular study. Br J Cancer 1987;55:437-442.

5 Jacobson RK, Lee KC, Muglia JJ, Robinson-Bostom L: Cutaneous manifestations of angioimmunoblastic T-cell lymphoma. J Am Acad Dermatol 2013;69:e25-e26.

6 Zhou Y, Attygalle AD, Chuang SS, Diss T, Ye H, Liu H, Hamoudi RA, Munson P, Bacon CM, Dogan A, Du MQ: Angioimmunoblastic T-cell lymphoma: histological progression associates with EBV and HHV6B viral load. Br J Haematol 2007;138:44-53.

7 Cogrel O, Beylot-Barry M, Vergier B, Dubus P, Doutre MS, Merlio JP, Beylot C: Sodium valproate-induced cutaneous pseudolymphoma followed by recurrence with carbamazepine. Br J Dermatol 2001;144:1235-1238.

8 Picard D, Janela B, Descamps V, D’Incan M, Courville P, Jacquot S, Rogez S, Mardivirin L, MoinsTeisserenc H, Toubert A, Benichou J, Joly P, Musette P: Drug reaction with eosinophilia and systemic symptoms (DRESS): a multiorgan antiviral T cell response. Sci Transl Med 2010;2:46ra62.

9 Singh A, Schabath R, Ratei R, Stroux A, Klemke CD, Nebe T, Flörcken A, van Lessen A, Anagnostopoulos I, Dörken B, Ludwig WD, Pezzutto A, Westermann J: Peripheral blood sCD3- CD4+ T cells: a useful diagnostic tool in angioimmunoblastic T cell lymphoma. Hematol Oncol 2014;32:16-21.

10 Cortés JR, Palomero T: The curious origins of angioimmunoblastic T-cell lymphoma. Curr Opin Hematol 2016;23:434-443. 


\section{Case Reports in Dermatology}

\begin{tabular}{l|l}
\hline Case Rep Dermatol 2017;9:74-79 \\
\hline DOI: 10.1159/000458752 & $\begin{array}{l}\text { (c) 2017 The Author(s). Published by S. Karger AG, Basel } \\
\text { www.karger.com/cde }\end{array}$ \\
\hline
\end{tabular}

Mangana et al.: Angioimmunoblastic T-Cell Lymphoma Mimicking Drug Reaction with Eosinophilia and Systemic Symptoms (DRESS Syndrome)
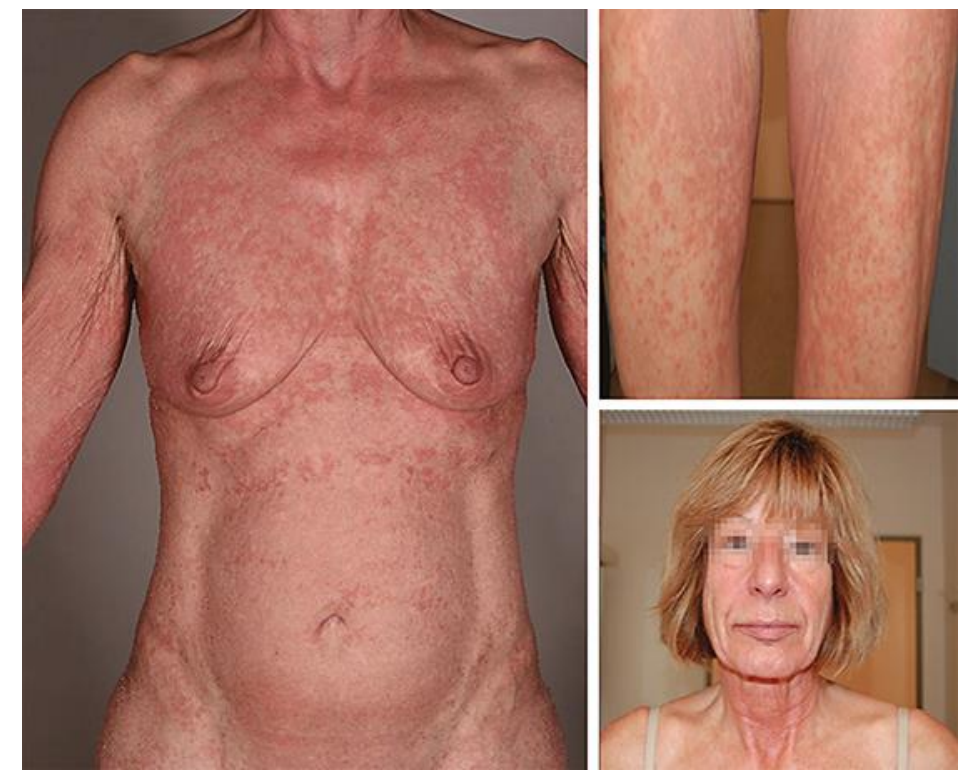

Fig. 1. Red confluent patches and plaques involving the trunk and extremities, including the face, with mild periorbital and facial edema.
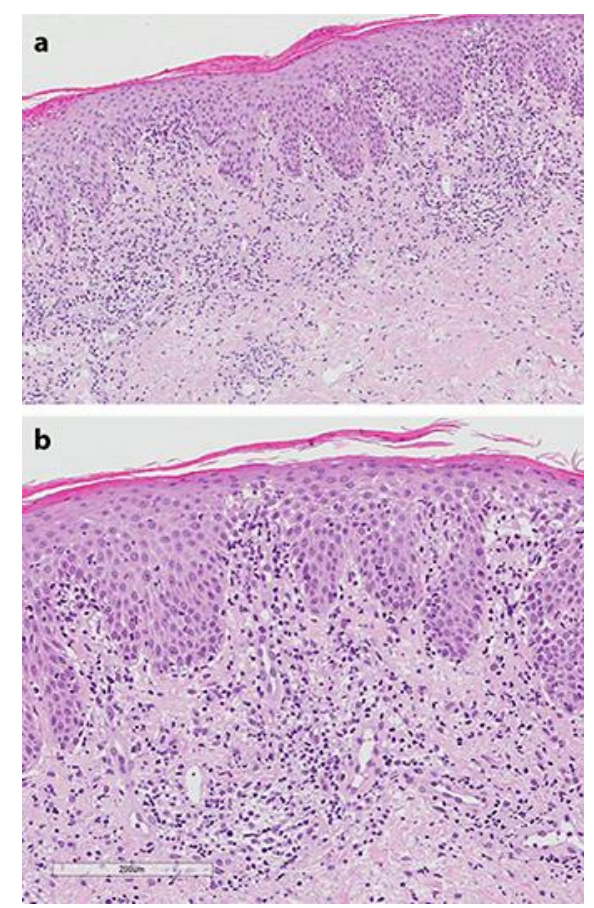

Fig. 2. a, b Skin biopsy showing spongiotic dermatitis with perivascular lymphocytic infiltration with eosinophils. 